\title{
CLEAN METAL NUCLEATED CASTING OF SUPERALLOYS
}

\author{
William T. Carter ${ }^{1}$, Joseph J. Jackson ${ }^{2}$, \\ Robin M. Forbes Jones ${ }^{3}$, Ramesh S. Minisandram ${ }^{3}$ \\ ${ }^{1}$ GE Global Research Center, 1 Research Circle, Niskayuna, NY 12309, USA \\ ${ }^{2}$ GE Energy, 300 Garlington Rd., Greenville, SC, 29615, USA \\ ${ }^{3}$ Allvac, 2020 Ashcraft Ave, Monroe, NC 28111, USA
}

Keywords: Casting, Electroslag Remelting, Nucleated Casting, Turbine Rotor

\begin{abstract}
The clean metal nucleated casting program is a cooperative research program between GE Energy and Allvac, sponsored by the National Institute of Standards and Technology under the Advanced Technology Program. The goal of the program is to develop a spray-casting technology for the production of extremely large, segregation-free, superalloy ingots for use in turbine wheels in land-based gas turbines. The raw material for the process is a superalloy vacuum induction melted (VIM) electrode; in the CMNC process it is melted in a bottompouring Electro-Slag Remelting (ESR) furnace that forms a stream of liquid superalloy for subsequent atomization and collection in a withdrawal mold. Gas atomization of the stream to form a spray occurs in a chamber where spray distance and gas-to-metal flow rates can be adjusted to cool the metal to a desired level before collection. The approach taken in the R\&D program is to address key technical risks associated with the system through construction of a one-ton research plant. These risks include the design and operability of the ESR furnace, pouring system, and collection system. Computational models of all key components of the process are developed and validated against experiments performed on the research plant. The validated models will be used to extrapolate to commercial-sized ingots.
\end{abstract}

\section{Introduction}

Conventional Turbine Rotor Production \& Need for Ingot Casting Innovation

The production of large gas turbine rotor forgings made from high temperature nickel base alloys such as Alloy 706 and Alloy 718 currently depends upon the successful processing of large diameter (up to $914 \mathrm{~mm}$ diameter) ingots which are subsequently billetized and forged. To achieve required chemistry control, homogeneity and freedom from ceramic inclusions, these large diameter starting ingots are triple melted using well established vacuum induction melting (VIM) to control chemistry, electro-slag remelting (ESR) to remove detrimental ceramics and vacuum arc remelting (VAR) to further refine and homogenize the ingot prior to thermomechanical working [1]. Improvements in process control have pushed the upper limit in production ingot diameters from $610 \mathrm{~mm}(24 \mathrm{in})$ in 1994 [2, 3], to $686 \mathrm{~mm}(27 \mathrm{in})$ in 1997 [4, 5], to $914 \mathrm{~mm}$ (36 in) in 2003 [6]. While these practices have successfully produced many Alloy 718 turbine rotor forgings currently operating in GE Energy gas turbine units, improvements in the reduction of potential segregation, grain size refinement and process simplification are desired to reduce product cost and enhance capability. This will especially be the case as more efficient larger turbine designs of the future will demand higher temperatures and stresses, requiring alloys even more complex than Alloy 718. CMNC offers a potential process path that can simplify 
processing, reduce segregation, and provide a finer grain input product for subsequent billetization and forging than possible today.

\section{The Clean Metal Nucleated Casting Process}

Clean metal nucleated casting $(\mathrm{CMNC})$ is a process that combines an oxide-free melting and pouring system that is based on electroslag remelting $[7,8]$, with the nucleated casting process $[9,10]$. The process is shown schematically in Figure 1. The raw material is a consumable electrode, which is cast from a vacuum induction melting system, and must be of the desired alloy chemistry. The goal of the CMNC process is to improve the quality of the metal by:

1. Removing any harmful oxide inclusions.

2. Removing any segregation defects or voids in the metal.

3. Reducing the grain size to allow efficient subsequent thermomechanical processing of the metal and/or increasing the diameter that can be cast in any particular alloy.

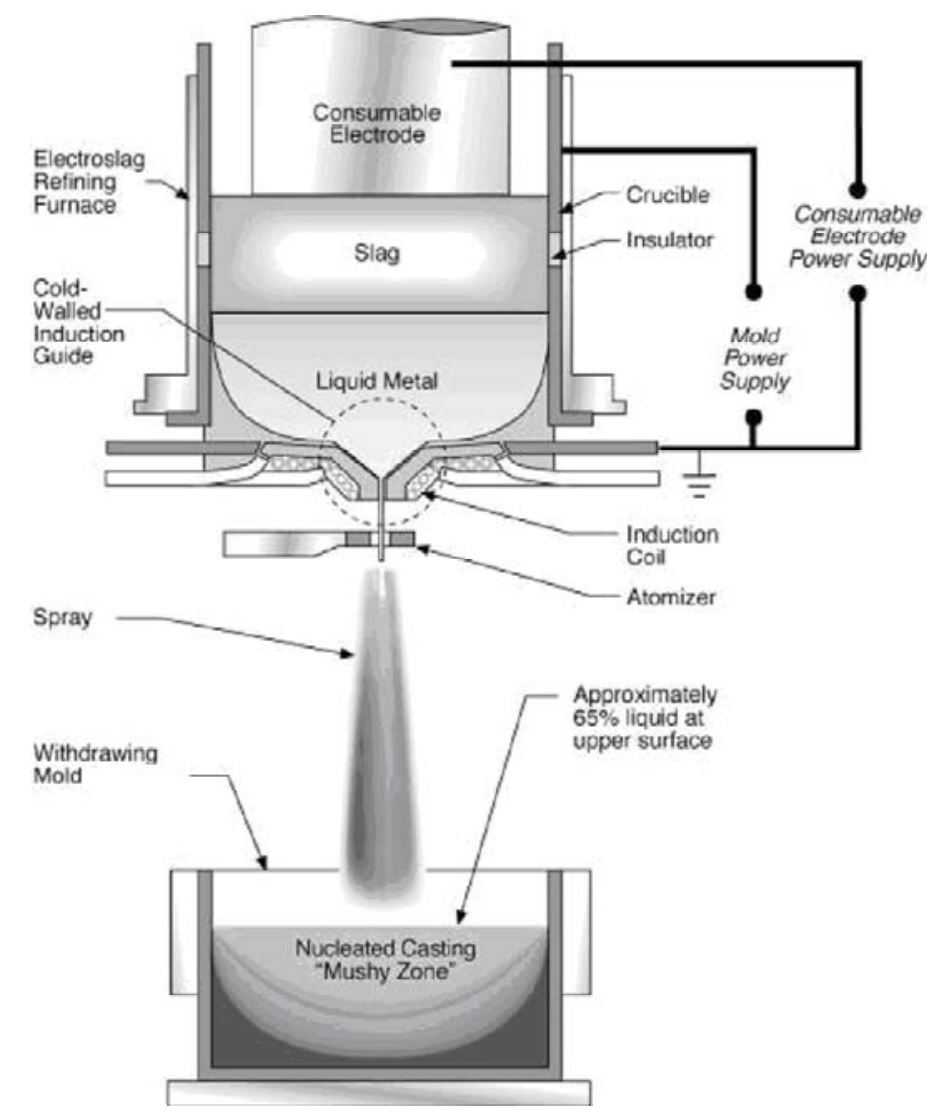

Figure 1. Clean metal nucleated casting process.

A description of the $\mathrm{CMNC}$ process sequence follows.

\section{$\underline{\text { ESR Melting System }}$}

A consumable electrode is fed into the furnace from above using a drive mechanism. The bottom face of the electrode is immersed in a hot liquid slag, which heats the bottom of the electrode causing it to melt. Metal droplets are formed on the face of the electrode and fall through the slag 
to form a liquid metal pool below the slag. Any oxide inclusions that are present in the electrode are exposed to the slag and are dissolved.

The slag is kept hot with alternating electric current, generally at low voltages and conventional frequencies, which is fed into the slag through the electrode. In most ESR facilities the required voltage is measured as a signal that is used to control the rate of advance of the electrode as the end is melted. Constant immersion depth is key for effective processing in conventional ESR melting, and various schemes for control of the voltage or voltage swing are in commercial use. These schemes are not required in the CMNC system because melting and solidification are decoupled as described below.

Alternatives exist for feeding electric current to the slag. In addition to the consumable electrode, current may be fed through an unconsumed electrode for additional process capability. An unconsumed electrode may take any of several geometric configurations $[11,12,13]$. In Figure 1 it is drawn as the top portion of the crucible. The unconsumed electrode must be made of a suitable material, or coated with a suitable material, to avoid contamination of the melt. The use of an unconsumed electrode allows decoupling to the furnace temperature from the melt rate; this decoupling is key to successful CMNC operation.

Two power supplies are indicated in Figure 1, one to feed the consumed electrode and one to feed the unconsumed electrode. It has been shown that this is not necessary, and a single power supply where both electrodes are wired in parallel, is sufficient. In fact, a measurement of the current in either leg of the circuit provides a useful feedback signal for control of a feed mechanism for advancing the consumed electrode.

\section{Pouring System}

The liquid metal is bottom-poured from the ESR furnace to form a liquid metal stream. To avoid contaminating the metal with oxide inclusions that may erode from a ceramic nozzle, a ceramicfree induction-heated copper funnel is used to form the stream. The copper nozzle is segmented radially and surrounded by an induction coil. Electric current is oscillated in the coil, inducing a current in each of the copper segments, subsequently inducing a heating current in the flowing liquid metal stream. Heat that is induced in the copper components is removed with cooling water. Experimentation with the copper nozzle, which has been termed a "Cold Induction Guide" (CIG), has shown the device to be approximately $10 \%$ efficient.

Significant effort is expended in conventional ESR processing to assure a constant melt rate because it assures a constant solidification rate, which has been shown to reduce the propensity to form segregation defects in ingots. In the CMNC process, the melting and the solidification processes are decoupled; it is the pour rate that must be held constant to assure a constant solidification rate. The pouring system relies on active pressure control above the melt to compensate for metal head changes that occur due to the integrated effect of any differences between melt rate and pour rate. Thus, a constant pour rate is maintained by measuring the melt level to control the pressure above the melt, requiring an enclosed melt chamber. The same signal is used to make adjustments to the furnace power, thereby influencing the melt rate to avoid over-filling or emptying the furnace.

\section{$\underline{\text { Atomization and Collection Systems }}$}

After a short free-fall from the CIG, the stream is atomized using a conventional open atomizer. This atomizer is similar to those used for spray forming, except that the gas to metal ratio is relatively low. The atomizer directs a gas jet onto the metal stream and converts it into a spray, 
accelerating the spray droplets from the atomization zone toward a collection mold, cooling them in flight. Nitrogen or argon can be used as the atomizing gas.

The process is tuned by adjusting the superheat of the metal stream, the gas-to-metal ratio, and the spray distance so as to obtain a metal pool with a top surface at approximately $25 \%-35 \%$ solid fraction. Thus, a semisolid metal is cast into the collection mold. When the particles strike the surface of the pool, large particles will be fully liquid, small particles will be fully solid, and medium sized particles will be semisolid. Many of the small solid particles will remelt after being deposited into the mold. However, when the system is tuned properly, a sufficient number of solid particles will remain to serve as nucleation site for further solidification. As these sites grow, they will eventually come into contact with adjacent growing zones, and solidification stops. If a sufficient number of nucleation sites are present, a fine-grained metallurgical structure results.

The water-cooled collection mold is key to the nucleated casting process. The CMNC process relies on a withdrawal mold with stationary sidewalls and a moveable bottom plate. As metal is deposited on the top of the ingot, the ingot is withdrawn through the stationary sidewalls so as to maintain a constant metal spray distance.

\section{Development of the CMNC Process}

\section{$\underline{\text { Status of Computational Modeling Activities }}$}

One of the key objectives of the CMNC project is to develop verified physics-based process models for use in process design and optimization in the commercialization phase.

As is evident from Figure 1, the process may be divided into several discrete stages, each of which may be modeled independently. The possible stages are:

1. Melting \& Pouring

2. Atomization

3. Spray

4. Ingot Casting

Of these, the physics of the atomization stage is very complex and deemed to be outside the scope of the present effort. Empirical information obtained via experimental observation will instead be used to describe atomization. Models are currently under development for all the other stages above.

ESR/CIG Model: An axisymmetric model has been developed for the electromagnetics, fluid flow and heat transfer in a coupled fashion. The present model incorporates alternating current in the electromagnetic calculations. Further, it incorporates the various current flow paths featured in the actual furnace. Figure 2 shows one such typical current path when the furnace is operating at $75 \%$ of its rated current capacity.

Experiments are ongoing for verification of this model. Once it has been verified, the model will be used to optimize furnace configuration and process parameters for various process requirements. 

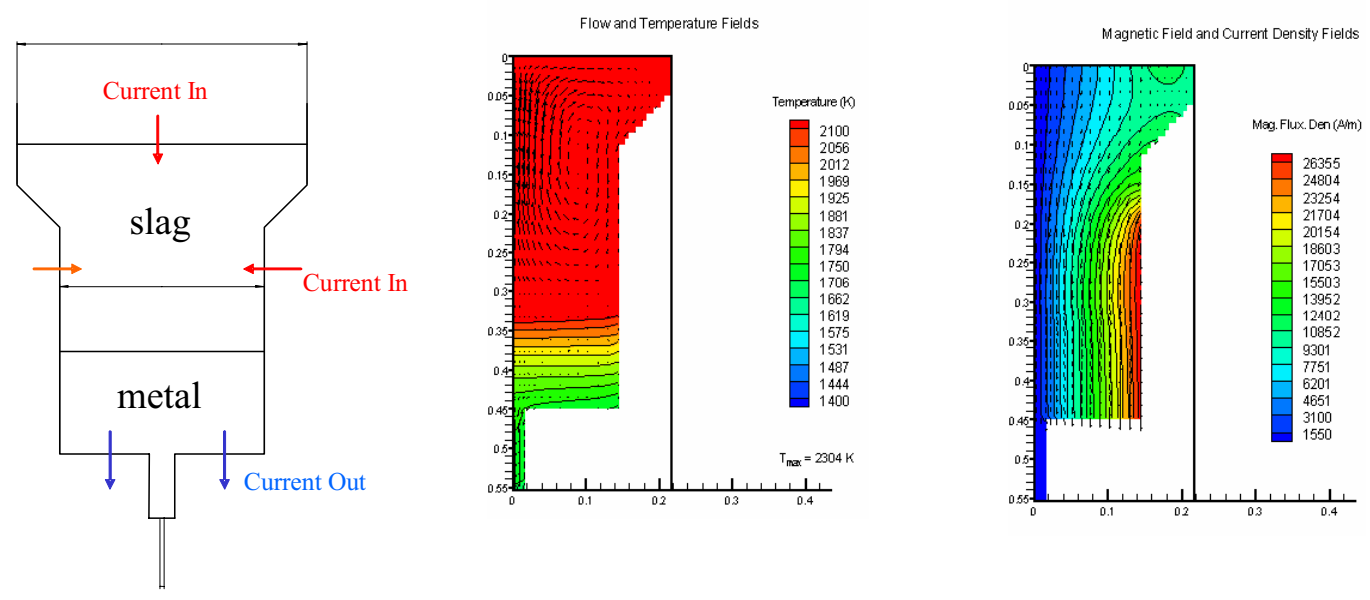

Figure 2. ESR/CIG model showing (left) a possible current configuration; (center) predicted temperature and flow fields when the furnace operates at $75 \%$ of rated amperage; and (right) the corresponding magnetic and current density fields.

2D Spray Model: The spray process is modeled by introducing a gas-metal mixture at one end of a computation zone (corresponding to a location some distance below the atomization zone, after atomization has been completed) and predicting how the velocities and temperature/solidification of the metal droplets in the spray evolve based on their interactions with the surrounding gas medium. Detailed experiments have been conducted to characterize the spray input condition under various operating parameters. For example, the particle size distribution has been measured at radial locations in one plane of the spray using the Phase Doppler Anemometry (PDA) technique. Figure 3 is a photograph of the spray during such a trial.

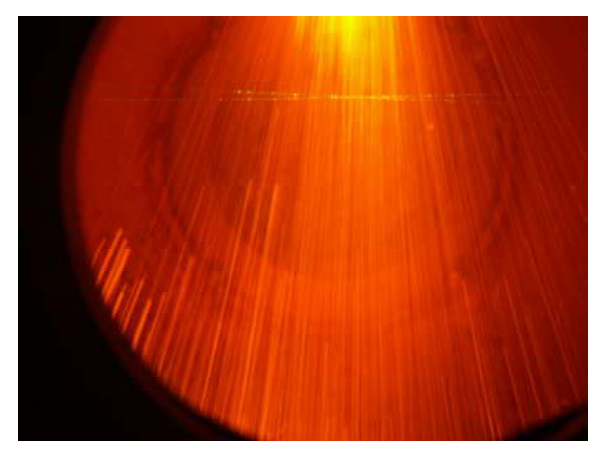

Figure 3. Particle size measurements of the spray are made using Phase Doppler Anemometry.

2D Chamber Model: An axisymmetric model incorporates the chamber geometry around the previously described 2D spray model. Its purpose is to determine the effect of chamber configuration on the flow and temperature of particles before they strike a collection mold. A typical geometry is shown in Figure 4. The corresponding results are shown in Figure 5, where the effect of the presence or absence of the overhang or lip in the schematic in Figure 4 is predicted. 


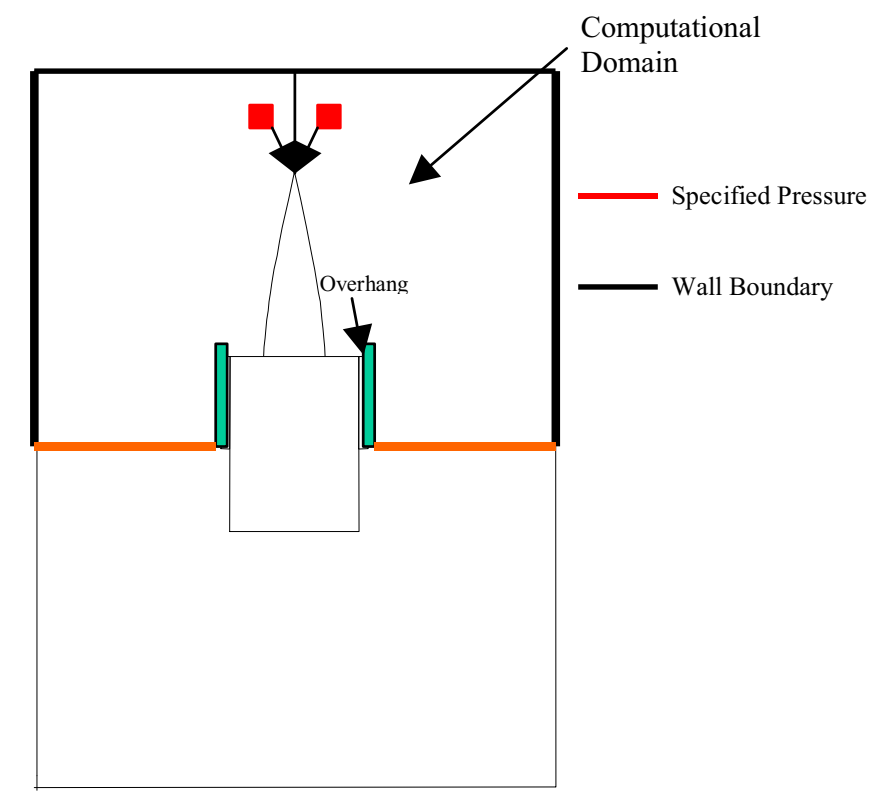

Figure 4. Typical chamber geometry.
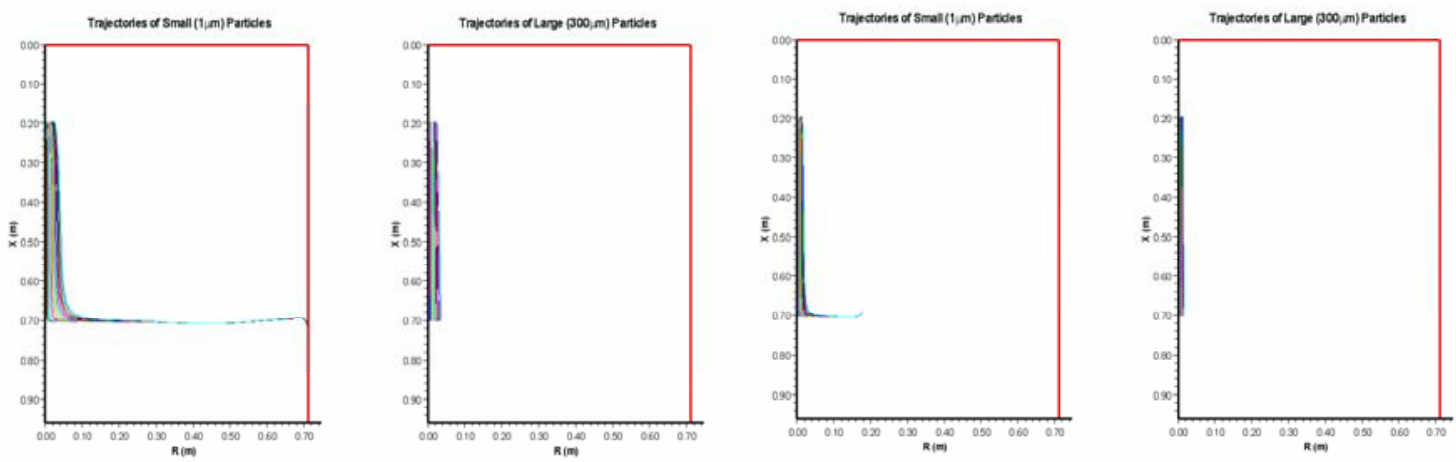

Figure 5. Results of analysis showing the effect of the overhang on particle flow. In the absence of the overhang (left), small particles ( $\sim 1$ micron) get carried away with the gas. When an overhang is present (right), these particles get trapped by the overhang and will fall into the deposit. Large particles ( $\sim 300$ microns) fall into the preform under the influence of gravity and the overhang has no influence on their travel.

Preform Model: This model is currently under development. The goal of the model is to predict metal flow in the mold for the purposes of filling the mold without casting defects. Key to success is the incorporation of a realistic material model for metal in the semi-solid state.

\section{$\underline{\text { Status of Subscale Trials }}$}

The sub-scale trials are aimed at understanding the effects of process variables on the deposition of the casting. Process variables such as metal superheat, pouring rate, atomizing gas pressure (gas to metal ratio), distance from the atomizer, rotation variables (speed and eccentricity) and spray variables (oscillation frequency) are being investigated. 
The trials are being conducted using 300 pound heats which are cast into an 18" diameter mold. The heats are melted in a vacuum induction furnace. Prior to casting, the chamber is backfilled with argon to equalize the pressure between the casting chamber and the melting chamber. The process window is assessed by correlating the deposition temperature with the appearance of the deposit as recorded by video tape-recorder along with an assessment of the structure and properties of the deposit.

The deposits are being evaluated by assessing the macrostructure and microstructure. Properties will be obtained from forged coupons taken from the deposit. To date, scoping experiments are being conducted to identify the limits of acceptable deposition parameters. Future work will concentrate on specific identification of acceptable process parameters.

\section{Conclusions}

The goal of the Clean Metal Nucleated Casting project is to extend the capabilities of superalloy casting to larger diameters, and more complex alloys, than possible today. The new process combines ESR with nucleated casting to cast a fine-grained, segregation-free ingot, without the limitations in size of triple melt ingots. The ongoing project includes a modeling effort coupled with pilot plant experimentation for the purposes of validating the models.

\section{Acknowledgements}

This project is partially sponsored by the U.S. National Institute of Standards and Technology under the Advanced Technology Program (No. 70NANB1H3042). The authors gratefully acknowledge their technical and financial support.

\section{References}

1. P.W. Schilke, J.J. Pepe, and R.C. Schwant, "Alloy 706 Metallurgy and Turbine Wheel Application," Superalloys 718, 625, 706 and Various Derivatives, The Minerals, Metals and Materials Society, Warrendale, PA, ed. E.A. Loria, 1993.

2. A. Mitchell, "Melting Processes and Solidification in Alloys 718-625," Superalloys 718, 625 and Various Derivatives, ed. E.A. Loria, The Minerals, Metals and Materials Society, 1991, 15-27.

3. A. Mitchell, "Progress in Understanding Clean Metal Production for IN718," Superalloys 718, 625 and Various Derivatives, ed. E.A. Loria, The Minerals, Metals and Materials Society, 1994, 109-124.

4. A.D. Helms, C.B. Adasczik, L.A. Jackman, "Extending the Size Limits of Cast/Wrought Superalloy Ingots," Superalloys 1996, ed. R.D. Kissinger, et.al., The Minerals Metals and Materials Society, 427-433.

5. R.C. Schwant, et.al., "Large 718 Forgings for Land Based Turbines," Superalloys 718 , 625 and Various Derivatives, E.A. Loria, Ed., 1997, 141-151.

6. Stephen J. Balsone, "Nickel-Base Superalloy Materials Technology for Advanced IGT Applications," International Symposium on Niobium for HT Application, CBMM - Companhia Brasileira de Metalurgia e Mineracao, Araxa, Brazil, December 1-3, 2003.

7. M.G. Benz and T.F. Sawyer, Direct Processing of Electroslag Refined Metal, U.S. Patent 5, 160,532, 1992. 
8. W.T. Carter, et.al, "Methods for flow control in electroslag refining process," US Patent 5, 649,992, 1997.

9. A. Sankaranarayanan, "Vertical Casting Process," U.S. Patent 5,381,847, 1995.

10. D.E. Tyler and W.G. Watson, "Nucleated Casting," Proceedings of the 1995 International Conference \& Exhibition on Powder Metallurgy and Particulate Materials, May 14-17, 1995, 7-99-7-107.

11. L.B. Medovar, "Electroslag Technology and Bi-Metallic Billets Including Rolling Mill Rolls," Proceedings of the 2002 International Symposium on Electroslag Remelting Technologies, Chicago, Oct. 20-22, 2002.

12. H. Holzgruber and W. Holzgruber, "New ESR Concepts for Improved Control of Solidification," Proceedings of the 2002 International Symposium on Electroslag Remelting Technologies, Chicago, Oct. 20-22, 2002.

13. W.S. Satya Prasad, et.al., "Recycling of Superalloy Scrap through Electroslag Refining," ISIJ Int. 36 (12), 1996, 1459-1464. 